\title{
Another walk through the world of chiral dynamics
}

\author{
Ulf-G. Meißner*† \\ Helmholtz-Institut für Strahlen- und Kernphysik and Bethe Center for Theoretical Physics \\ Universität Bonn, D-53115 Bonn, Germany \\ Institute for Advanced Simulation, Institut für Kernphysik and Jülich Center for Hadron Physics, \\ Forschungszentrum Jülich, D-52425 Jülich, Germany \\ Tbilisi State University, 0186 Tbilisi, Georgia \\ E-mail: meissner@hiskp.uni-bonn.de
}

Chiral dynamics is a pretty mature field. Nonetheless, there are many exciting new developments. In this opening talk, I consider S-wave, isospin-zero pion-pion scattering and the calculation of the width of the lightest baryon resonances at two loops. New insights into the chiral dynamics of charm-strange mesons are discussed as well as recent results on the flavor decomposition of the pion-nucleon $\sigma$-term. I end with a short wish-list of lattice QCD tests pertinent to chiral dynamics.

The 9th International Workshop on Chiral Dynamics,

September $17-21,2018$

Duke University, Durham, North Carolina, USA

\footnotetext{
*Speaker.

${ }^{\dagger}$ Work supported in part by DFG \& NSFC (CRC 110), CAS PIFI (2018DM0034), and VolkswagenStiftung (93652)
} 


\section{Introduction}

Chiral dynamics is a pretty mature field. We have high-precision calculations and tests in the $\pi \pi$ and $\pi N$ systems, that is in the two-flavor sector of the light up and down quarks. This is largely based on a fruitful marriage of chiral perturbation theory (CHPT) with dispersion theory. In case of pion-pion scattering, such a scheme also allows one to pin down precisely the parameters of the lightest resonances in QCD. There is also much progress in lattice QCD. However, especially in the sector with baryon number $B \geq 1$, there is still a lack of calculations of scattering processes, not even to talk about precision. The situation in the three-flavor sector remains ambiguous as $m_{s} \sim \Lambda_{\mathrm{QCD}}$. Still, there is some marked progress in eta and kaon decays. When it comes to baryons, the most urgently needed calculation concerns the flavor-singlet piece of the pion-nucleon $\sigma$-term, $\sigma_{0}$, as I will discuss later. In this context, the lattice can and does provide numbers on the strange matrix element $m_{s}\langle p|\bar{s} s| p\rangle$, which nicely complements the dispersive determination of $\sigma_{\pi N}$. Furthermore, unitarization schemes offer insight into excited states, though at some cost. In often used approximations, the analytical structure is not always respected, so improvements of the method for coupled channels are required. Nevertheless, there has been some remarkable progress in heavy-light systems combining unitarized CHPT, lattice QCD results and accurate data. In what follows, I give more details on some of these statements but the reader is advised to consult the many fine talks given at this workshop for further discussions and references.

\section{Lesson 1: Once more on the $\pi \pi$ scattering length $a_{0}$}

Elastic pion-pion scattering $(\pi \pi \rightarrow \pi \pi)$ is the purest process in two-flavor chiral dynamics as the up and the down quark masses are really small compared to any other hadronic mass scale. At threshold the scattering amplitude is given in terms of two numbers, the scattering lengths $a_{0}$ and $a_{2}$. In my 2012 talk [1], I had reviewed the experimental and the theoretical status, concluding: "This is truly one of the finest tests of the Standard Model at low energies. However, not all is well - a direct lattice determination of $a_{0}$ is still missing and the lattice practitioners are urged to provide this so important number. Such a calculation is, of course, technically challenging because of the disconnected diagrams, but time is ripe for doing it." Since then, only two groups $[2,3]$ have published results on this so important quantity, summarized in Tab. 1. Both of these are consistent with the high-precision result from Roy equations, $a_{0}=0.220 \pm 0.005$ [4], but presumably underestimate the systematic errors. There have also been very nice lattice calculations concerning the $\mathrm{S}$-wave, isospin-zero $\pi \pi$ phase shifts and the $f_{0}(500)$ (sigma) meson [5, 6], but these do not give values for $a_{0}$. To get a better handle on the disconnected diagrams, connected and dis-

\begin{tabular}{|lccc|}
\hline Author(s) & $a_{0}$ & Fermion action & Pion mass range \\
\hline Fu [2] & $0.214(4)(7)$ & asqtad staggered & $240-430 \mathrm{MeV}$ \\
Liu et al.[3] & $0.198(9)(6)$ & twisted mass & $250-320 \mathrm{MeV}$ \\
\hline
\end{tabular}

Table 1: Lattice QCD determinations of $a_{0}$.

connected contractions in $\pi \pi$ scattering have been analyzed in Ref. [7]. There are four types of contractions, namely the connected ones, called direct (D) and crossed (C) contractions, the singly 

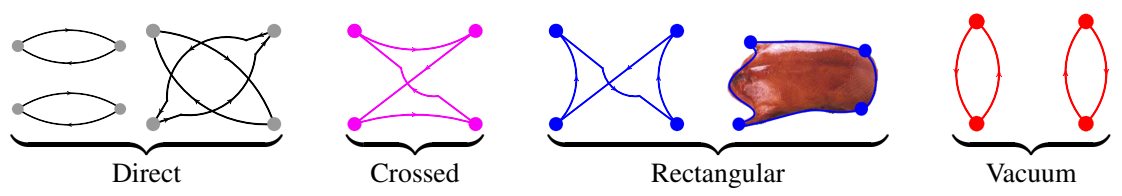

Figure 1: Types of Wick contractions in pion-pion scattering.

disconnected ones of rectangular (or roasted Peking duck ${ }^{1}$ ) type $(\mathrm{R})$ and the doubly disconnected vacuum graphs $(\mathrm{V})$, which are most difficult to calculate in lattice $\mathrm{QCD}$, cf. Fig. 1. By means of partially-quenched chiral perturbation (PQCHPT) theory [8, 9, 10, 11], one can distinguish and analyze the effects from different types of contraction diagrams to the pion-pion scattering amplitude, in particular also to $a_{0}$. The findings of Ref. [7] can be summarized as follows: The R and C-type diagrams are leading order (LO) in the chiral and the large- $N_{C}$ expansion, see also [12], whereas the $\mathrm{D}$ - and V-type diagrams are next-to-leading order (NLO) in the chiral and the large- $N_{C}$ expansion. As expected, the R-type diagram dominates when it contributes (as is the case for $a_{0}$ ), while neglecting the V-type contribution reduces $a_{0}$ by about $12 \%$.

This program was recently carried further in Ref. [13]. One has to make a direct connection to the energy levels measured in lattice QCD. This can be achieved in the following way: First, each contraction in $\pi \pi$ scattering can be represented by a physical scattering process between two pions in SU(4|2) partially quenched QCD. Then, the multi-channel scattering matrix can be diagonalized to obtain four effective single-channel scattering amplitudes. Two of these (labeled $\alpha, \beta$ ) involve only connected contractions and the others (called $\gamma, \delta$ ) contain disconnected pieces. With that, one can immediately relate the threshold parameters of each single-channel scattering amplitude to the corresponding discrete energy levels in a finite volume through the usual single-channel Lüscher formula. Further, the threshold parameters can be expressed in terms of physical and unphysical LECs in the SU(4|2) PQCHPT, which can be fitted to discrete energy levels extracted from the connected $\pi \pi$ correlation functions. In particular, fitting to certain data form the European Twisted Mass Collaboration [14], one achieves an order-of-magnitude improvement over the earlier result [15] in the determination of the LECs combination $3 L_{0}^{\mathrm{PQ}, r}+L_{3}^{\mathrm{PQ}, r}$. Performing lattice computations using more volumes, one can also improve the precision of the worst known LEC $L_{0}^{\mathrm{PQ}, r}$. With these fitted LECs one is able to predict the discrete energy shifts $\delta E_{0}^{\gamma, \delta}$, which involve disconnected contractions, as functions of the lattice size. For more details, see [13].

\section{Lesson 2: The width of the lightest baryon resonances}

In this section, I will be concerned with calculating the width of the two lightest baryon resonances, the $\Delta(1232)$ and the Roper $N^{*}(1440)$. This might at first sight appear irritating, as imaginary parts are usually not precisely reproduced in CHPT. For that simple reason, one has to employ a complex-mass scheme and work to two loops.

Consider first the width of the $\Delta$ at two-loop order [16]. The pertinent effective Lagrangian contains, besides many other terms, the leading $\pi \Delta$ and $\pi N \Delta$ couplings, parameterized in terms of

\footnotetext{
${ }^{1}$ As introduced by Feng-Kun Guo in 2017, see also Fig. 1 that was generated by him.
} 


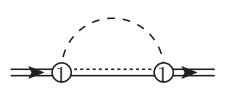

(a)

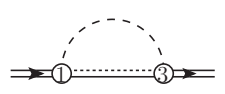

(f)

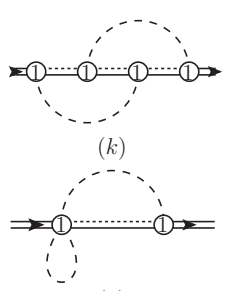

$(p)$

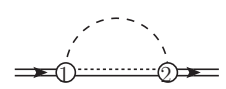

(b)

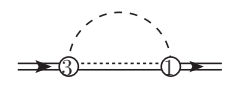

(g)
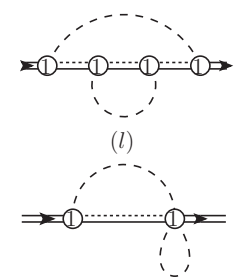

$(q)$

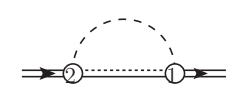

(c)

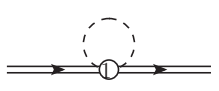

(h)

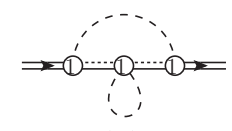

$(m)$

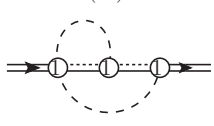

$(r)$

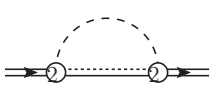

(d)

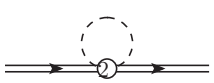

(i)

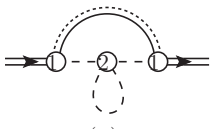

$(n)$

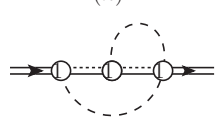

(s)

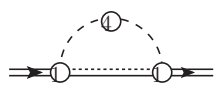

(e)

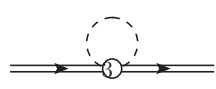

$(j)$

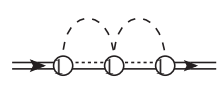

(o)

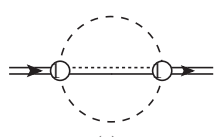

$(t)$

Figure 2: One and two-loop self-energy diagrams contributing to the width of the delta resonance up-to-andincluding fifth order according to the standard power counting. The dashed and double solid lines represent the pions and the delta resonances, respectively. The double (solid-dotted) lines in the loops correspond to either nucleons or deltas. The numbers in the circles give the chiral orders of the vertices.

the LECs $g_{1}$ and $h$, respectively,

$$
\begin{aligned}
\mathscr{L}_{\pi \Delta}^{(1)} & =-\bar{\Psi}_{\mu}^{i} \xi_{i j}^{\frac{3}{2}}\left\{\left(i \not D^{j k}-m_{\Delta} \delta^{j k}\right) g^{\mu v}-i\left(\gamma^{\mu} D^{v, j k}+\gamma^{v} D^{\mu, j k}\right)+i \gamma^{\mu} \not D^{j k} \gamma^{v}\right. \\
& \left.+m_{\Delta} \delta^{j k} \gamma^{\mu} \gamma^{v}+g_{1} \frac{1}{2} y^{j k} \gamma_{5} g^{\mu v}+g_{2} \frac{1}{2}\left(\gamma^{\mu} u^{v, j k}+u^{v, j k} \gamma^{\mu}\right) \gamma_{5}+g_{3} \frac{1}{2} \gamma^{\mu} \mu^{j k} \gamma_{5} \gamma^{v}\right\} \xi_{k l}^{\frac{3}{2}} \Psi_{v}^{l}, \\
\mathscr{L}_{\pi N \Delta}^{(1)} & =h \bar{\Psi}_{\mu}^{i} \xi_{i j}^{\frac{3}{2}} \Theta^{\mu \alpha}\left(z_{1}\right) \omega_{\alpha}^{j} \Psi_{N}+\text { h.c. },
\end{aligned}
$$

in terms of the spin-1/2 (3/2) fields $\Psi_{N}\left(\Psi_{\mu}^{i}\right)$ and for other notations, see [16]. The power counting rests on $m_{\Delta}-m_{N}$ being a small quantity. However, there are so many LECs (not all are shown in Eq. (3.1)), so how can one one possibly make a prediction? The trick is to use the complex-mass renormalization scheme, a method that was originally introduced for precision $W, Z$-physics, see e.g. $[17,18]$ and later transported to chiral EFT [19]. For the case under consideration, we need to evaluate the $\Delta$ self-energy on the complex pole,

$$
z-m_{\Delta}^{0}-\Sigma(z)=0 \quad \text { with } \quad z=m_{\Delta}-i \frac{\Gamma_{\Delta}}{2} .
$$

The corresponding diagrams for the one- and two-loop self-energy contributing to the width of the delta resonance up to order $q^{5}$ are displayed in Fig. 2, where the counterterm diagrams are not shown. The one-loop diagrams are easily obtained, for the calculation of the two-loop graphs one uses the Cutkovsky rules for instable particles, that relate the width to the pion-nucleon scattering amplitude, $\Gamma_{\Delta} \sim|A(\Delta \rightarrow N \pi)|^{2}$ [20]. One finds a remarkable reduction of parameters that is reflected in the relation

$$
\begin{aligned}
h_{A} & =h-\left(b_{3} \Delta_{23}+b_{8} \Delta_{123}\right)-\left(f_{1} \Delta_{23}+f_{2} \Delta_{123}\right) \Delta_{123}+2\left(2 f_{4}-f_{5}\right) M_{\pi}^{2}, \\
\Delta_{23} & =m_{N}-m_{\Delta}, \quad \Delta_{123}=\left(M_{\pi}^{2}+m_{N}^{2}-m_{\Delta}^{2}\right) /\left(2 m_{N}\right),
\end{aligned}
$$




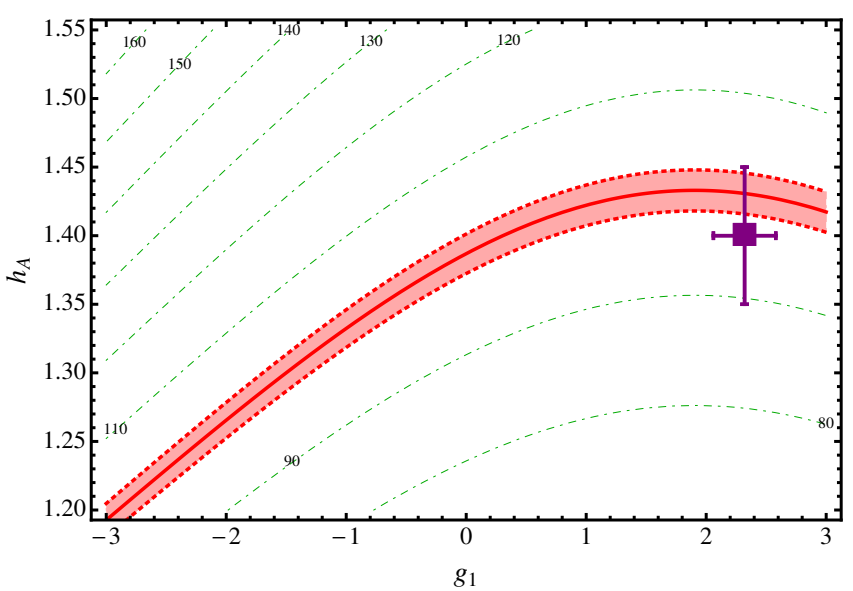

Figure 3: Correlation between the leading $\pi \Delta$ and $\pi N \Delta$ couplings. The band gives the uncertainty of the calculation, and the box with the error bars are the results from the analysis of Ref. [21].

which means that all of the LECs appearing in the $\pi N \Delta$ interaction at second and third order, the $b_{i}(i=3,8)$ and $f_{i}(i=1,2,4,5)$, respectively, merely lead to a renormalization of the LO $\pi N \Delta$ coupling $h$, and, consequently, one finds a very simple formula for the decay width $\Delta \rightarrow N \pi$,

$$
\Gamma(\Delta \rightarrow N \pi)=\left(53.91 h_{A}^{2}+0.87 g_{1}^{2} h_{A}^{2}-3.31 g_{1} h_{A}^{2}-0.99 h_{A}^{4}\right) \mathrm{MeV} .
$$

This leads to a novel correlation, that is independent of the number of colors, as depicted in Fig. 3. It is obviously fulfilled by the analyis of Ref. [21], that showed that the inclusion of the $\Delta$ alleviates the tension between the threshold and subthreshold regions in the description of $\pi N$ scattering found in baryon CHPT.

Next, I consider the calculation of the width of the Roper $N^{*}(1440)$ at two-loop order [22]. A remarkable feature of the Roper is the fact that its decay width into a nucleon and a pion is similar to the width into a nucleon and two pions. Any model that is supposed to describe the Roper must account for this fact. In CHPT, consider the effective chiral Lagrangian of pions, nucleons and deltas $[23,24,25]$,

$$
\begin{aligned}
\mathscr{L}_{\mathrm{eff}} & =\mathscr{L}_{\pi \pi}+\mathscr{L}_{\pi N}+\mathscr{L}_{\pi \Delta}+\mathscr{L}_{\pi R}+\mathscr{L}_{\pi N \Delta}+\mathscr{L}_{\pi N R}+\mathscr{L}_{\pi \Delta R}, \\
\mathscr{L}_{\pi R}^{(1)} & =\bar{\Psi}_{R}\left\{i \not D-m_{R}+\frac{1}{2} g_{R} \psi l \gamma^{5}\right\} \Psi_{R}, \quad \mathscr{L}_{\pi R}^{(2)}=\bar{\Psi}_{R}\left\{c_{1}^{R}\langle\chi+\rangle\right\} \Psi_{R}+\ldots, \\
\mathscr{L}_{\pi N R}^{(1)} & =\bar{\Psi}_{R}\left\{\frac{1}{2} g_{\pi N R} \gamma^{\mu} \gamma_{5} u_{\mu}\right\} \Psi_{N}+\text { h.c. }, \mathscr{L}_{\pi \Delta R}^{(1)}=h_{R} \bar{\Psi}_{\mu}^{i} \xi_{i j}^{\frac{3}{2}} \Theta^{\mu \alpha}(\tilde{z}) \omega_{\alpha}^{j} \Psi_{R}+\text { h.c. },
\end{aligned}
$$

where the leading Roper-pion, Roper-nucleon pion and Delta-Roper-pion couplings, $g_{R}, g_{\pi N R}$ and $h_{R}$, respectively, are high-lighted. In this case, the power counting is complicated, but can be set up around the complex pole as [22]:

$$
m_{R}-m_{N} \sim \varepsilon, m_{R}-m_{\Delta} \sim \varepsilon^{2}, m_{\Delta}-m_{N} \sim \varepsilon^{2}, M_{\pi} \sim \varepsilon^{2},
$$

where $\varepsilon$ denotes a small parameter. Again, let us calculate the self-energy to two loops at the complex pole $z_{R}=m_{R}-i \Gamma_{R} / 2$. By applying the cutting rules to these self-energy diagrams, one 
obtains the graphs contributing to the decay amplitudes of the Roper resonance into the $\pi N$ and $\pi \pi N$ systems, leading to the total width

$$
\Gamma_{R}=\Gamma_{R \rightarrow N \pi}+\Gamma_{R \rightarrow N \pi \pi}
$$

A somewhat lengthy calculation leads to:

$$
\begin{aligned}
\Gamma(R \rightarrow N \pi)= & 550(58) g_{\pi N R}^{2} \mathrm{MeV} \\
\Gamma(R \rightarrow N \pi \pi)= & \left(1.49(0.58) g_{A}^{2} g_{\pi N R}^{2}-2.76(1.07) g_{A} g_{\pi N R}^{2} g_{R}\right. \\
& +1.48(0.58) g_{\pi N R}^{2} g_{R}^{2}+2.96(0.94) g_{A} g_{\pi N R} h_{A} h_{R} \\
& \left.-3.79(1.37) g_{\pi N R} g_{R} h_{A} h_{R}+9.93(5.45) h_{A}^{2} h_{R}^{2}\right) \mathrm{MeV} .
\end{aligned}
$$

The total width thus depends on five LECs. The uncertainties in the round brackets are generated by the uncertainties in the LECs. We use $g_{A}=1.27$ and $h_{A}=1.42 \pm 0.02$. The latter value is the real part of this coupling taken from Ref. [26]. As for the other unknown parameters, the authors of [22] fixed $g_{\pi N R}$ so as to reproduce the width $\Gamma_{R \rightarrow \pi N}=(123.5 \pm 19.0) \mathrm{MeV}$ from the PDG. This yields $g_{\pi N R}= \pm(0.47 \pm 0.04)$. In what follows, let us take the positive sign for our central value and use the negative one as part of the error budget. Further, assume $g_{R}=g_{A}$ and $h_{R}=h_{A}$, the socalled maximal mixing assumption [27]. Then, one can make a prediction for the two-pion decay width of the Roper,

$$
\Gamma(R \rightarrow N \pi \pi)=\left(41 \pm 22_{\mathrm{LECs}} \pm 17_{\mathrm{h} . \mathrm{o} .}\right) \mathrm{MeV},
$$

which is consistent with the PDG value of $(67 \pm 10) \mathrm{MeV}$. The error due to the neglect of the higher orders (h.o.) is simply given by multiplying the $\varepsilon^{5}$ result (central value) with $\varepsilon=\left(m_{R}-m_{N}\right) / m_{N} \simeq$ 0.43. Clearly, to make further progress, we need an improved determination of the LECs $g_{R}$ and $h_{R}$.

\section{Lesson 3: New insights into heavy-light meson spectroscopy}

In 2012, I had already considered scattering the Goldstone boson octet $(\pi, K, \eta)$ off the $D$ meson triplet $\left(D^{0}, D^{+}, D_{s}^{+}\right)$. This involves the positive-parity scalar charm-strange mesons $D_{s 0}^{*}(2317)$ and $D_{s 1}(2460)$, which are very narrow and are often interpreted as molecular $D K$ and $D^{*} K$ states, respectively. Over the years, a number of puzzles has emerged that need to be answered: (1) why are the masses of the $D_{s 0}^{\star}(2317)$ and the $D_{s 1}(2460)$ much lower than the quark model predictions for $c \bar{s}$ mesons? (2) why is $M_{D_{s 1}(2460)}-M_{D_{s 0}^{\star}(2317)} \simeq M_{D^{\star}}-M_{D}$ within $2 \mathrm{MeV}$ ? and (3) why does one find $M_{D_{0}^{\star}(2400)} \gtrsim M_{D_{s 0}^{\star}(2317)}$ and $M_{D_{1}(2430)} \simeq M_{D_{s 1}(2460)}$ ? Naively, one would expect that the mesons, where a light $(u, d)$ quark is substituted by an $s$ quark, are heavier. Within the molecular picture, the combination of unitarized CHPT, lattice results and precision data has led to a consistent picture, which I will only briefly discuss here. For more details the reader should consult the comprehensive review [28].

Concerning the first puzzle: It is resolved within the molecular scenario, as known since long. This is further supported by recent lattice QCD calculations with $c \bar{s}$ and $D K$ interpolating fields and relatively light pions [29, 30]. The $D K$ scattering length from Ref. [29], $a_{0}=-1.33(20) \mathrm{fm}$, is consistent with the prediction of the molecular scenario, $a_{0}^{\text {mol }}=-2 / \sqrt{\mu_{D K} \varepsilon} \simeq-1 \mathrm{fm}$, with 
$\mu_{D K}$ the reduced mass and $\varepsilon \simeq 45 \mathrm{MeV}$ the binding energy. Further, the quark mass dependence of the $D_{s 0}^{\star}(2317)$ calculated in Ref. [30] is perfectly consistent with the one in the molecular picture, cf. Ref. [31]. The wavefunction renormalization constant deduced from these results, $1-Z=1.04(0.08)(0.30)$ is also supporting the molecular scenario in which $Z^{\mathrm{mol}}=0$. The second puzzle is also naturally explained in this picture as a natural consequence of Heavy Quark Spin Symmetry (HQSS), one simply substitutes the $D$-meson by a $D^{*}$-meson, see e.g. [32]. Using Heavy Quark Flavor Symmetry (HQFS), one can furthermore easily make predictions for the bottom analogues, $M_{B_{s 0}^{\star}} \simeq M_{B}+M_{K}-45 \mathrm{MeV} \simeq 5.730 \mathrm{GeV}$ and $M_{B_{s 1}} \simeq M_{B^{\star}}+M_{K}-45 \mathrm{MeV} \simeq 5.776 \mathrm{GeV}$, which are consistent with lattice QCD, $M_{B_{s 0}^{\star}}^{\text {lat }}=(5.711 \pm 0.013 \pm 0.019) \mathrm{GeV}$ and $M_{B_{s 1}}^{\text {lat }}=(5.750 \pm$ $0.017 \pm 0.019) \mathrm{GeV}$ [33]. Finally, let me resolve enigma (3). Recently, the Hadron Spectrum Collaboration investigated coupled channel $D \pi, D \eta$ and $D_{s} \bar{K}$ scattering with $I=1 / 2$ and found one pole corresponding to the $D_{0}^{\star}(2400)$ [33]. These lattice energy levels were re-analyzed in Ref. [34] using unitarized CHPT and the values of the LECs already determined long before in [35]. They found two poles, completely analogous to the famous $\Lambda(1405)$, where the lighter one has mass of about $2.1 \mathrm{GeV}$, thus resolving puzzle (3). This pattern of two $I=1 / 2$ states emerges naturally in the underlying formalism since already the LO CHPT interactions are attractive in two flavor multiplets, to which the two states belong: the anti-triplet and the sextet [36, 34]. This double-pole structure had already been noticed in earlier calculations, see e.g. [36, 37] but only now finds a natural explanation. Using HQSS and HQFS, one can extend this double-pole scenario to the $D_{1}, B_{0}^{\star}$ and $B_{1}$, as summarized in Tab. 2 .

\begin{tabular}{|cccc|}
\hline & Lower Pol $[\mathrm{MeV}]$ & Higher Pol $[\mathrm{MeV}]$ & PDG $[\mathrm{MeV}]$ \\
\hline$D_{0}^{\star}$ & $\left(2105_{-8}^{+6}, 102_{-11}^{+10}\right)$ & $\left(2451_{-26}^{+36}, 134_{-8}^{+7}\right)$ & $(2318 \pm 29,134 \pm 20)$ \\
$D_{1}$ & $\left(2247_{-6}^{+5}, 107_{-10}^{+11}\right)$ & $\left(2555_{-30}^{+47}, 203_{-9}^{+8}\right)$ & $\left(2427 \pm 40,192_{-55}^{+65}\right)$ \\
$B_{0}^{\star}$ & $\left(5535_{-11}^{+9}, 113_{-17}^{+15}\right)$ & $\left(5852_{-19}^{+16}, 36 \pm 5\right)$ & - \\
$B_{1}$ & $\left(5584_{-11}^{+9}, 119_{-17}^{+14}\right)$ & $\left(5912_{-18}^{+15}, 42_{-4}^{+5}\right)$ & - \\
\hline
\end{tabular}

Table 2: Two-pole scenario in various $I=1 / 2$ states in the heavy meson sector. Given are the mass $M$ and the half-width $\Gamma / 2$ as $(M, \Gamma / 2)$.

Remarkable, there is further support for this picture from the recent high precision results for $B \rightarrow D \pi \pi$ from LHCb [38]. They determined accurately the so-called angular moments from the $D \pi$ final-state interactions (FSI) that contain spectroscopic information. The corresponding chiral Lagrangian for $\bar{B} \rightarrow D$ transition with the emission of two light pseudoscalars is known since long [39]. Combining it with the FSI from unitarized CHPT, one gets a two parameter description of the corresponding amplitude in the energy region of the $D \pi$ system below $2.5 \mathrm{GeV}$, $\mathscr{A}\left(B^{-} \rightarrow D^{+} \pi^{-} \pi^{-}\right)=\mathscr{A}_{0}(s)+\mathscr{A}_{1}(s)+\mathscr{A}_{2}(s)$, in terms of S-, P- and D-waves. In Ref. [40] the same $\mathrm{P}$ - and $\mathrm{D}$-waves as in the $\mathrm{LHCb}$ analysis were taken and the $\mathrm{S}$-wave was fixed form the coupled $D \pi, D \eta, D_{s} \bar{K}$ system already determined before. The two parameters are one combination of LECs of the aforementioned chiral Lagrangian and the subtraction constant in the two-meson loop functions $G_{i j},\{i j\} \in\left\{D \pi, D \eta, D_{s} \bar{K}\right\}$. The angular moments are well reproduced, see Fig. 4. This picture can further be tested by confrontation with data, namely by measuring the angular moments, in particular $\left\langle P_{1}\right\rangle-14\left\langle P_{3}\right\rangle / 9$, with unprecedented accuracy for the $B \rightarrow D^{(*)} \pi \pi$ and $B \rightarrow D_{s}^{(*)} \bar{K} \pi$ reactions. This can be done at $\mathrm{LHCb}$ and Belle-II. One expects to see nontrivial cusp structures at 

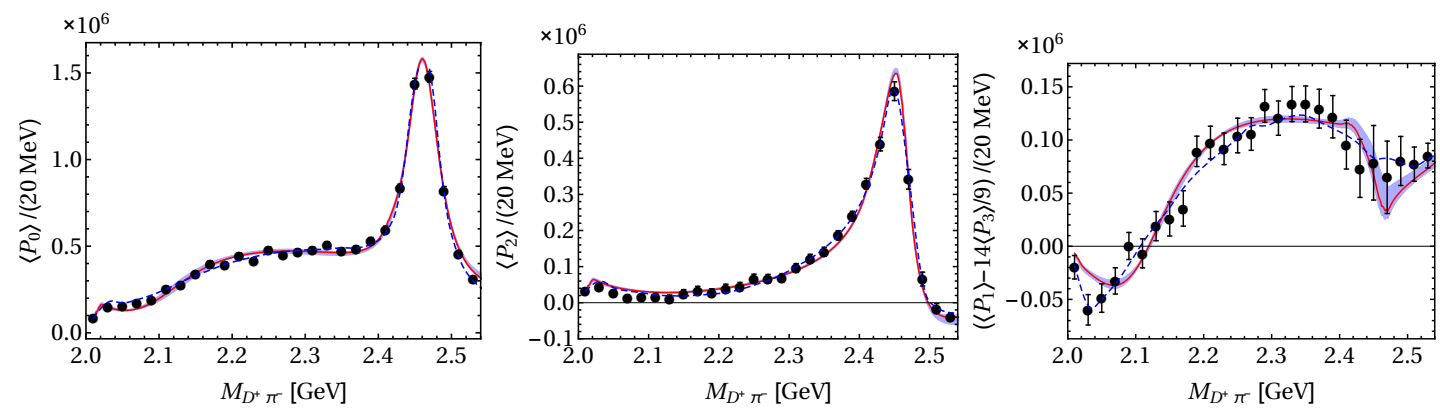

Figure 4: Fit to the LHCb data for the angular moments $\left\langle P_{0}\right\rangle,\left\langle P_{1}\right\rangle-14\left\langle P_{3}\right\rangle / 9$ and $\left\langle P_{2}\right\rangle$ for the $B^{-} \rightarrow$ $D^{+} \pi^{-} \pi^{-}$reaction [38]. The largest error among $\left\langle P_{1}\right\rangle$ and $14\left\langle P_{3}\right\rangle / 9$ in each bin is taken as the error of $\left\langle P_{1}\right\rangle-14\left\langle P_{3}\right\rangle / 9$. The solid lines show the results of [40], with error bands corresponding to the one-sigma uncertainties propagated from the input scattering amplitudes, while the dashed lines stand for the LHCb fit using cubic splines for the $S$-wave.

the $D^{(*)} \eta$ and $D_{s}^{(*)} \bar{K}$ thresholds in the former, and near-threshold enhancement in the $D_{s}^{(*)} \bar{K}$ spectrum in the latter [34]. Also, measuring the hadronic width of the $D_{s 0}^{*}(2317)$, predicted to be about $100 \mathrm{keV}$ in the molecular scenario [41, 35], while much smaller otherwise. This is a smoking-gun type of experiment and will be performed by the PANDA experiment at FAIR. Finally, one can check the existence of the sextet pole in lattice QCD with a relatively large SU(3) symmetric quark mass. And finally, one should search for the predicted analogous bottom positive-parity mesons both experimentally and in lattice QCD.

I therefore conclude that the long accepted paradigm underlying open-flavor heavy meson spectroscopy that identifies all ground states with $c \bar{q}$ or $b \bar{q}$ quark model states, is no longer tenable. In a broader view, the hadron spectrum must be viewed as more than a collection of quark model states, but rather as a manifestation of a more complex dynamics that leads to an intricate pattern of various types of states that can only be understood by a joint effort from experiment, lattice QCD and phenomenology.

\section{Lesson 4: A short remark on the pion-nucleon $\sigma$-term}

The situation concerning the Roy-Steiner analysis to precisely determine the pion-nucleon $\sigma$ term, $\sigma_{\pi N}$, and the apparent tension with lattice determinations has been nicely reviewed by Martin Hoferichter [42], see also the impressive work by the BMWc Collaboration reported by Laurent Lellouch [43]. One new piece of information concerns the flavor singlet term $\sigma_{0}$, related to $\sigma_{\pi N}$ and the strangeness fraction $y$ via $\sigma_{\pi N}=\sigma_{0} /(1-y)$. In Ref. [44] the flavor decomposition of $\sigma_{\pi N}$ was re-analyzed in the framework of baryon chiral perturbation to fourth order, employing both a covariant (EOMS) and the heavy baryon framework and including also the low-lying decuplet (for the different approaches, see e.g. the review [45]). Due to the tension between the lattice and RoySteiner determinations, only continuum data were used, making the fits at fourth order difficult due to the large number of LECs. Within some Bayesian approach, one arrives at the results collected in Tab. 3. At third order, one finds a disturbingly large spread within the different approaches. Similar results for the $\mathscr{O}\left(p^{3}\right)$ fits have been obtained earlier in [46]. The $\mathscr{O}\left(p^{4}\right)$ results for the covariant calculation only differ slightly from the $\mathscr{O}\left(p^{3}\right)$ results. The fit without decuplet-resonances shifts 


\begin{tabular}{|c|c|c|c|c|}
\hline & HB & HB + decuplet & EOMS & EOMS + decuplet \\
\hline $\mathscr{O}\left(p^{3}\right)$ & $57.9(0.2)(17.0)$ & $88.6(0.2)(34.0)$ & $46.4(0.2)(10.4)$ & $57.6(0.2)(17.0)$ \\
$\mathscr{O}\left(p^{4}\right)$ & $64.1(31.7)(9.3)$ & $64.0(31.7)(18.7)$ & $51.8(31.4)(5.7)$ & $61.8(31.4)(9.3)$ \\
\hline
\end{tabular}

Table 3: Results for $\sigma_{0}$ in $\mathrm{MeV}$ at third and fourth order. Here, "+decuplet" means the inclusion of the decuplet, HB denotes the heavy baryon and EOMS the covariant approach. The first error comes from the uncertainties within the given order, the second error is an estimate of the neglected higher order effects following Ref. [47].

more towards $\sigma_{\pi N} \simeq 59 \mathrm{MeV}$, while the fit with the decuplet is slightly above $60 \mathrm{MeV}$. The $\mathscr{O}\left(p^{4}\right)$ HB fit results are both around $64 \mathrm{MeV}$. So the $\mathscr{O}\left(p^{4}\right)$ result for the $\mathrm{HB}$ approach including decuplet baryons is much closer to $\sigma_{\pi N}$ than its corresponding $\mathscr{O}\left(p^{3}\right)$ result. Given the central values for $\sigma_{0}$ from Tab. 3 and the precise value from the Roy-Steiner analysis for $\sigma_{\pi N}$ [48], we see that the strangeness content $y \simeq 0$, but due to the large uncertainties, this can not be made more precise.

\section{Short summary \& outlook}

Picking up on my 2012 talk, it is obvious that hadron-hadron scattering continues to be a prime playground for chiral dynamics. Over the years, a nice interplay of (U)CHPT, dispersion relations, lattice QCD and experiment has developed, as discussed before in a few examples. There is a clear challenge to lattice QCD, namely the precise determinations of the scattering lengths $a_{0}$ in $\pi \pi \rightarrow \pi \pi, a_{0}^{1 / 2}, a_{0}^{3 / 2}$ in $\pi K \rightarrow \pi K$ and $a^{+}, a^{-}$in $\pi N \rightarrow \pi N$. This would be premier tests of the chiral QCD dynamics. Also, the latter are particularly important for another test of the lattice QCD determinations of $\sigma_{\pi N}[49]$.

Furthermore, there has been recent focus on heavy-light systems, where the marriage of CHPT with Heavy Quark Effective Theory and dispersive techniques starts to pay large dividends. Also, (heavy) hadron decays play an ever increasing role, largely due the large number of scattering processes with light mesons in the final states that are otherwise difficult to access experimentally. On the continuum side, complex mass renormalization schemes and dispersion relations appear most fruitful, while on the lattice side there is some focus on the development of finite-volume formulas for three-body decays, see e.g. Ref. [50] (and references therein). Clearly, we need $\omega \rightarrow 3 \pi$ and $R \rightarrow N \pi \pi$ from lattice QCD.

Finally, as nicely demonstrated by Evgeny Epelbaum in his talk [51], chiral symmetry plays an indispensable role in nuclei. I look forward to many existing new results in the years to come.

\section{Acknowledgements}

I thank all my collaborators for sharing their insight into the topics discussed here.

\section{References}

[1] U.-G. Meißner, “A walk through the world of chiral dynamics,” PoS CD 12 (2013) 012.

[2] Z. Fu, "Lattice QCD study of the s-wave $\pi \pi$ scattering lengths in the $\mathrm{I}=0$ and 2 channels," Phys. Rev. D 87 (2013) 074501. 
[3] L. Liu et al., "Isospin-0 $\pi \pi$ s-wave scattering length from twisted mass lattice QCD," Phys. Rev. D 96 (2017) 054516.

[4] G. Colangelo, J. Gasser and H. Leutwyler, “The pi pi S wave scattering lengths," Phys. Lett. B 488 (2000) 261.

[5] R. A. Briceno, J. J. Dudek, R. G. Edwards and D. J. Wilson, "Isoscalar $\pi \pi$ scattering and the $\sigma$ meson resonance from QCD,” Phys. Rev. Lett. 118 (2017) 022002.

[6] D. Guo, A. Alexandru, R. Molina, M. Mai and M. Döring, "Extraction of isoscalar $\pi \pi$ phase-shifts from lattice QCD,” Phys. Rev. D 98 (2018) 014507.

[7] N. R. Acharya, F. K. Guo, U.-G. Meißner and C. Y. Seng, "Connected and disconnected contractions in pion-pion scattering," Nucl. Phys. B 922 (2017) 480.

[8] C. W. Bernard and M. F. L. Golterman, "Partially quenched gauge theories and an application to staggered fermions," Phys. Rev. D 49 (1994) 486.

[9] S. R. Sharpe and N. Shoresh, "Physical results from unphysical simulations," Phys. Rev. D 62 (2000) 094503.

[10] S. R. Sharpe and N. Shoresh, "Partially quenched chiral perturbation theory without Phi0," Phys. Rev. D 64 (2001) 114510.

[11] C. Bernard and M. Golterman, "On the foundations of partially quenched chiral perturbation theory," Phys. Rev. D 88 (2013) 014004.

[12] F. K. Guo, L. Liu, U.-G. Meißner and P. Wang, “Tetraquarks, hadronic molecules, meson-meson scattering and disconnected contributions in lattice QCD,” Phys. Rev. D 88 (2013) 074506.

[13] N. R. Acharya, F. K. Guo, U.-G. Meißner and C. Y. Seng, "Constraints on Disconnected Contributions in $\pi \pi$ Scattering," arXiv:1902.10290 [hep-lat].

[14] C. Helmes et al. [ETM Collaboration], "Hadron-hadron interactions from $\mathrm{N}_{f}=2+1+1$ lattice QCD: isospin-2 $\pi \pi$ scattering length,” JHEP 1509 (2015) 109.

[15] P. A. Boyle et al., "Low energy constants of SU(2) partially quenched chiral perturbation theory from $\mathrm{N}_{f}=2+1$ domain wall QCD,” Phys. Rev. D 93 (2016) 054502.

[16] J. Gegelia, U.-G. Meißner, D. Siemens and D. L. Yao, "The width of the $\Delta$-resonance at two loop order in baryon chiral perturbation theory," Phys. Lett. B 763 (2016) 1.

[17] R. G. Stuart, in "Z0 Physics”, ed. J. Tran Thanh Van (Editions Frontieres, Gif-sur-Yvette, 1990), p. 41.

[18] A. Denner, S. Dittmaier, M. Roth and D. Wackeroth, "Predictions for all processes e+ e- $\longrightarrow 4$ fermions + gamma," Nucl. Phys. B 560 (1999) 33 doi:10.1016/S0550-3213(99)00437-X [hep-ph/9904472].

[19] D. Djukanovic, J. Gegelia, A. Keller and S. Scherer, "Complex-mass renormalization in chiral effective field theory,” Phys. Lett. B 680 (2009) 235

[20] M. J. G. Veltman, "Unitarity and causality in a renormalizable field theory with unstable particles," Physica 29 (1963) 186.

[21] D. Siemens, J. Ruiz de Elvira, E. Epelbaum, M. Hoferichter, H. Krebs, B. Kubis and U.-G. Meißner, "Reconciling threshold and subthreshold expansions for pion-nucleon scattering," Phys. Lett. B 770 (2017) 27. 
[22] J. Gegelia, U.-G. Meißner and D. L. Yao, "The width of the Roper resonance in baryon chiral perturbation theory," Phys. Lett. B 760 (2016) 736.

[23] B. Borasoy, P. C. Bruns, U.-G. Meißner and R. Lewis, "Chiral corrections to the Roper mass," Phys. Lett. B 641 (2006) 294.

[24] D. Djukanovic, J. Gegelia and S. Scherer, "Chiral structure of the Roper resonance using complex-mass scheme,” Phys. Lett. B 690 (2010) 123

[25] B. Long and U. van Kolck, "The Role of the Roper in Chiral Perturbation Theory," Nucl. Phys. A 870-871 (2011) 72

[26] D. L. Yao, D. Siemens, V. Bernard, E. Epelbaum, A. M. Gasparyan, J. Gegelia, H. Krebs and U.-G. Meißner, "Pion-nucleon scattering in covariant baryon chiral perturbation theory with explicit Delta resonances," JHEP 1605, 038 (2016).

[27] S. R. Beane and U. van Kolck, “The Role of the Roper in QCD,” J. Phys. G 31 (2005) 921.

[28] F. K. Guo, C. Hanhart, U.-G. Meißner, Q. Wang, Q. Zhao and B. S. Zou, "Hadronic molecules,” Rev. Mod. Phys. 90 (2018) 015004.

[29] D. Mohler, C. B. Lang, L. Leskovec, S. Prelovsek and R. M. Woloshyn, " $D_{s 0}^{*}(2317)$ Meson and D-Meson-Kaon Scattering from Lattice QCD,” Phys. Rev. Lett. 111 (2013) 222001.

[30] G. S. Bali, S. Collins, A. Cox and A. Schäfer, "Masses and decay constants of the $D_{s 0}^{*}(2317)$ and $D_{s 1}(2460)$ from $N_{f}=2$ lattice QCD close to the physical point," Phys. Rev. D 96 (2017) 074501.

[31] M. L. Du, F. K. Guo, U.-G. Meißner and D. L. Yao, "Study of open-charm $0^{+}$states in unitarized chiral effective theory with one-loop potentials,” Eur. Phys. J. C 77 (2017) 728.

[32] M. Cleven, F. K. Guo, C. Hanhart and U.-G. Meißner, "Light meson mass dependence of the positive parity heavy-strange mesons," Eur. Phys. J. A 47 (2011) 19.

[33] C. B. Lang, D. Mohler, S. Prelovsek and R. M. Woloshyn, "Predicting positive parity B $_{s}$ mesons from lattice QCD,” Phys. Lett. B 750 (2015) 17.

[34] M. Albaladejo, P. Fernandez-Soler, F. K. Guo and J. Nieves, "Two-pole structure of the $D_{0}^{*}(2400)$," Phys. Lett. B 767 (2017) 465.

[35] L. Liu, K. Orginos, F.-K. Guo, C. Hanhart and U.-G. Meißner, "Interactions of Charmed Mesons with Light Pseudoscalar Mesons from Lattice QCD and Implications on the Nature of the $D_{s 0}^{*}(2317)$," Phys. Rev. D 87 (2013) 014508.

[36] E. E. Kolomeitsev and M. F. M. Lutz, “On Heavy light meson resonances and chiral symmetry,” Phys. Lett. B 582 (2004) 39.

[37] F. K. Guo, P. N. Shen, H. C. Chiang, R. G. Ping and B. S. Zou, "Dynamically generated 0+ heavy mesons in a heavy chiral unitary approach,” Phys. Lett. B 641 (2006) 278.

[38] R. Aaij et al. [LHCb Collaboration], “Amplitude analysis of $B^{-} \rightarrow D^{+} \pi^{-} \pi^{-}$decays," Phys. Rev. D 94 (2016) 072001.

[39] M. J. Savage and M. B. Wise, “SU(3) Predictions for Nonleptonic B Meson Decays,” Phys. Rev. D 39 (1989) 3346.

[40] M. L. Du, M. Albaladejo, P. Fernandez-Soler, F. K. Guo, C. Hanhart, U.-G. Meißner, J. Nieves and D. L. Yao, “Towards a new paradigm for heavy-light meson spectroscopy," Phys. Rev. D 98 (2018) 094018. 
[41] M. F. M. Lutz and M. Soyeur, "Radiative and isospin-violating decays of D(s)-mesons in the hadrogenesis conjecture," Nucl. Phys. A 813 (2008) 14.

[42] Martin Hoferichter, these proceedings.

[43] Laurent Lellouch, these proceedings.

[44] D. Severt, U.-G. Meißner and J. Gegelia, "Flavor decomposition of the pion-nucleon $\sigma$-term," arXiv:1902.10508 [hep-ph].

[45] V. Bernard, “Chiral Perturbation Theory and Baryon Properties,” Prog. Part. Nucl. Phys. 60 (2008) 82.

[46] J. M. Alarcon, L. S. Geng, J. Martin Camalich and J. A. Oller, “The strangeness content of the nucleon from effective field theory and phenomenology,” Phys. Lett. B 730 (2014) 342.

[47] E. Epelbaum, H. Krebs and U.-G. Meißner, "Improved chiral nucleon-nucleon potential up to next-to-next-to-next-to-leading order,” Eur. Phys. J. A 51 (2015) 53.

[48] M. Hoferichter, J. Ruiz de Elvira, B. Kubis and U.-G. Meißner, "High-Precision Determination of the Pion-Nucleon $\sigma$ Term from Roy-Steiner Equations,” Phys. Rev. Lett. 115 (2015) 092301.

[49] M. Hoferichter, J. Ruiz de Elvira, B. Kubis and U.-G. Meißner, "Remarks on the pion-nucleon $\sigma$-term," Phys. Lett. B 760 (2016) 74.

[50] M. Döring, H.-W. Hammer, M. Mai, J.-Y. Pang, A. Rusetsky and J. Wu, "Three-body spectrum in a finite volume: the role of cubic symmetry," Phys. Rev. D 97 (2018) 114508.

[51] Evgeny Epelbaum, these proceedings. 